\title{
Importance of monitoring arsenic methylation metabolism in acute promyelocytic leukemia patients receiving the treatment of arsenic trioxide
}

Yu Zheng ${ }^{\dagger}$, Yuan-Fei Mao ${ }^{\dagger}$, Hui-Jin Zhao ${ }^{\dagger}$, Li Chen, Li-Ning Wang, Yun-Xiang Zhang, Jiong Hu, Jun-Min Li ${ }^{*}$, Xiao-Yang Li ${ }^{*}$ and Hong-Ming Zhu*

\begin{abstract}
Background: Arsenic trioxide [ATO, inorganic arsenite (iAs $\left.{ }^{\prime \prime \prime}\right)$ in solution] plays an important role in the treatment of acute promyelocytic leukemia (APL). However, the long-term adverse effects (AEs) and the retention of arsenic among APL patients are rarely reported. In this study, we focused on arsenic methylation metabolism and its relationship with chronic hepatic toxicity, as we previously reported, among APL patients who had finished the treatment of ATO.

Methods: A total of 112 de novo APL patients who had completed the ATO-containing treatment were enrolled in the study. Arsenic species [iAs ${ }^{\prime \prime \prime}$, inorganic arsenate $\left(i A s^{V}\right)$, and their organic metabolites, monomethylarsonic acid (MMA) and dimethylarsinic acid (DMA)] in patients' plasma, urine, hair and nails were detected by high-performance liquid chromatography combined with inductively coupled plasma mass spectrometry (HPLC-ICP-MS). Eighteen single nucleotide polymorphisms (SNPs) of the arsenic ( +3 oxidative state) methylation transferase (AS3MT) gene, which was known as the main catalyzer for arsenic methylation, were tested with the polymerase chain reaction method.

Results: The study showed the metabolic pattern of arsenic in APL patients undergoing and after the treatment of ATO, in terms of total arsenic (TAs) and four species of arsenic. TAs decreased to normal after 6 months since cessation of ATO. But the arsenic speciation demonstrated significantly higher portion of iAs $s^{\text {II }}$ in patient's urine (40.08\% vs. $1.94 \%, P<0.001)$, hair $(29.25 \%$ vs. $13.29 \%, P=0.002)$ and nails $(30.21 \%$ vs. $13.64 \%, P=0.003)$ than the healthy controls', indicating a decreased capacity of arsenic methylation metabolism after the treatment of ATO. Urine primary methylation index (PMI) was significantly lower in patients with both chronic liver dysfunction $(0.14 \mathrm{vs} .0 .28, P=0.047)$ and hepatic steatosis ( 0.19 vs. $0.3, P=0.027)$, suggesting that insufficient methylation of arsenic might be related to chronic liver disorders. Two SNPs (A9749G and A27215G) of the AS3MT gene were associated with impaired urine secondary methylation index (SMI).
\end{abstract}

\footnotetext{
*Correspondence: ljm10378@rjh.com.cn; Ixy11811@rjh.com.cn;

zhm11931@rjh.com.cn

†Yu Zheng, Yuan-Fei Mao and Hui-Jin Zhao contributed equally to this

work

Shanghai Institute of Hematology, State Key Laboratory of Medical

Genomics, National Research Center for Translational Medicine

at Shanghai, Ruijin Hospital Affiliated to Shanghai Jiao Tong University

School of Medicine, No. 197 Rui Jin Er Road, Shanghai 200025, China
}

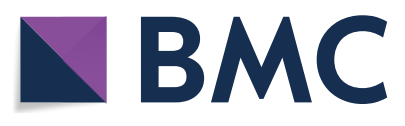

(c) The Author(s) 2021. This article is licensed under a Creative Commons Attribution 4.0 International License, which permits use, sharing, adaptation, distribution and reproduction in any medium or format, as long as you give appropriate credit to the original author(s) and the source, provide a link to the Creative Commons licence, and indicate if changes were made. The images or other third party material in this article are included in the article's Creative Commons licence, unless indicated otherwise in a credit line to the material. If material is not included in the article's Creative Commons licence and your intended use is not permitted by statutory regulation or exceeds the permitted use, you will need to obtain permission directly from the copyright holder. To view a copy of this licence, visit http://creativeco mmons.org/licenses/by/4.0/. The Creative Commons Public Domain Dedication waiver (http://creativecommons.org/publicdomain/ zero/1.0/) applies to the data made available in this article, unless otherwise stated in a credit line to the data. 
Conclusions: The long-term follow-up of arsenic speciation indicated a decreased arsenic methylation metabolism and a probable relationship with chronic hepatic disorders among APL patients after the cessation of ATO. Urine PMI could be a monitoring index for chronic AEs of ATO, and the SNPS of AS3MT gene should be considered when determining the dosage of ATO.

Keywords: Arsenic trioxide, Arsenic speciation, Arsenic methylation metabolism

\section{Background}

Arsenic trioxide (ATO) has achieved great success in the treatment of acute promyelocytic leukemia (APL) in combination with all-trans retinoic acid (ATRA), improving the rate of complete remission (CR) up to $92 \%$ or higher, [1-3] and the long-term overall survival (OS) reaching 87-97\% [4-8]. However, the retention and metabolism of ATO in patients are unclear, and the potential long-term arsenic intoxication and secondary carcinogenesis of ATO are rarely reported.

Arsenic speciation by high-performance liquid chromatography and inductively coupled plasma mass spectrometry (HPLC-ICP-MS) has uncovered the pattern of arsenic metabolism recently in the field of toxicology and environmentology. It is now known that the total arsenic (TAs) is composed of inorganic arsenics (iAs) and organic arsenics, and +3 and +5 oxidative state inorganic arsenics, i.e. iAs ${ }^{\mathrm{III}}$ (as in ATO and $\mathrm{As}_{2} \mathrm{~S}_{3}$ ) and iAs ${ }^{\mathrm{V}}$ (as in $\mathrm{As}_{2} \mathrm{O}_{5}$ ), are the most toxic species [9]. The methylation metabolism of arsenic is considered as the detoxification process $[9,10]$. Inorganic arsenics are methylated in the hepatocytes into less toxic metabolites, such as monomethylarsonic acid (MMA) and dimethylarsinic acid (DMA), and excreted via urine. Extra iAs is accumulated in the skin, hair and nails due to the high affinity of iAs ${ }^{\text {III }}$ to the sulfydryl ($\mathrm{SH})$ in the cytokeratin. The urine primary methylation index $\left[\mathrm{PMI}=\mathrm{MMA} /\left(\mathrm{iAs}^{\mathrm{III}}+\mathrm{iAs}^{\mathrm{V}}\right)\right]$ and the secondary methylation index $(\mathrm{SMI}=\mathrm{DMA} / \mathrm{MMA})$ [11] are recommended to measure the level of arsenic methylation in human body. High urine PMI or SMI represents relatively sufficient metabolism with little iAs retention. Moreover, an $S$-adenosyl methionine dependent arsenic $(+3$ oxidative state) methylation transferase (AS3MT) in the hepatocyte is crucial in the arsenic methylation metabolism [12]. Recent studies also showed that some single nucleotide polymorphisms (SNPs) on the exons or introns of $A S 3 M T$ gene might affect the variations in individual's ability of arsenic metabolism. The most commonly accepted SNP was the M287T heterozygote on the exon, which was related to better methylation capacity. Other SNPs on the introns (including G12390C, T12590C, T35587C, T14215C, G35991A and so on) were also reported to be associated with elevated DMA or MMA in the urine [13-16].
We have long been focused on the chronic adverse effects (AEs) and retention of ATO in APL patients with ATRA and ATO combination therapy. In the initial report with 5-year follow-up, Hu et al. [1] showed that the concentration of arsenic in patients' plasma, urine, hair and nails after the treatment decreased significantly compared to patients undergoing ATO treatment, only slightly higher than healthy controls, while no chronic AEs or secondary tumor were observed. Later we reported $15.2 \%$ of chronic liver dysfunction and $42.9 \%$ of hepatic steatosis (both $\mathrm{P}<0.001$ compared to healthy controls) in a 12-year follow-up. But the concentration of TAs in patients' plasma, urine, hair and nails after cessation of ATO were no higher than healthy controls [5]. In an attempt to explore the relationship between chronic hepatic toxicity and the exposure to ATO, in this study we further analyzed the arsenic methylation metabolism by arsenic speciation and its related genes among these patients.

\section{Methods \\ Patients and treatment protocol}

Between January 2001 and June 2012, a total of 112 patients with newly diagnosed APL who had completed the whole treatment were retrospectively enrolled in this study, and they were all in persistent molecular remission by the last follow-up. Criteria of diagnosis, cytogenetic analysis and regular reverse transcription-polymerase chain reaction of $P M L-R A R A$ transcripts were performed as previously described [17]. All patients received the ATRA and ATO combination therapy according to the protocol as previously reported, [1,17] namely ATRA and ATO (plus anthracyclines if the white blood cell count was over $10 \times 10^{9} / \mathrm{L}$ ) in the induction phase, three courses of chemotherapy in the consolidation, and five cycles of ATRA, ATO and low-dose chemotherapy sequential treatment in the maintenance therapy. The dosage of ATO was 5 to $10 \mathrm{mg}$ daily for 28 days per cycle. Patients with persistent remission were treated with ATO for a total of 6 cycles, thus the total dose of ATO for all patients were $840 \mathrm{mg}$ to $1680 \mathrm{mg}$.

\section{Arsenic speciation}

The blood, urine, hair and nail samples of 112 patients, 112 healthy controls and 7 APL patients undergoing 
ATO treatment (as positive controls) were collected with informed consent. The study was proved by the Ethics Committee of Ruijin Hospital in Shanghai, China (approval number 2012-48 and 2013-22). Samples of positive controls were collected at 4 time points: before ATO (d0), after 7 days of ATO administration (d7), when achieving CR (usually collected 10-14 days after the induction therapy) and at the end of consolidation therapy (Con, including 3 courses of consolidation therapy with 28 days of ATO in each course). Plasma from the centrifuged blood samples and urine samples were reserved at $-80{ }^{\circ} \mathrm{C}$. Hair samples taken $2 \mathrm{~cm}$ near the scalp and fingernails of ten fingers were collected and stored at room temperature shielded from light before testing. All the biological samples were precisely prepared before the test of arsenic speciation as follows:

Plasma One tube of the plasma $(1-1.5 \mathrm{~mL})$ was mixed with $5 \mathrm{~mL}$ of methanol and water mixture (3:1) and extracted overnight on an oscillator twice. All centrifuged supernatant was mixed together and dried with 99.999\% nitrogen, then dissolved with $2 \mathrm{~mL}$ ultra-pure water.

Urine One tube of the urine sample (about $10 \mathrm{~mL}$ ) was centrifuged before filtered by Sep Pak C18 disposable extraction column (Tianjin Agela Technologies Co., Ltd.) and $0.45 \mu \mathrm{m}$ filter (Sangon Biotech Co., Ltd.), and diluted for four times.

Hair and nails The hair and nail samples were washed according to the "Trace element pollutants washing step" by International Atomic Energy Agency (IAEA) protocol (Ryabukhin YS, Al-Sharistani H. Activation analysis of hair as an indicator of contamination of man by environmental trace element pollutants. Vienna: International Atomic Energy Agency (IAEA); 1978. Report No.: IAEA/ $R L / 50)$, then dried and cut into pieces $(<2 \mathrm{~mm})$. Hair or nail pieces were mixed with $5 \mathrm{~mL}$ ultra-pure water and bathed at $90{ }^{\circ} \mathrm{C}$ for $6 \mathrm{~h}$ for extraction. The samples were centrifuged twice, and the supernatant was reserved for arsenic speciation.

Certified standard material, including $\mathrm{AsO}_{3}{ }^{3-}$ for iAs ${ }^{\mathrm{III}}$, $\mathrm{AsO}_{4}{ }^{3-}$ for iAs ${ }^{\mathrm{V}}, \mathrm{CH}_{3} \mathrm{AsO}_{3}{ }^{2-}$ for MMA and $\mathrm{C}_{2} \mathrm{H}_{7} \mathrm{AsO}_{2}$ for DMA, were purchased from Chinese Institute of Metrology (numbers of certification were GBW08666, GBW08667, GBW08668, and GBW08669, respectively). Ultra-pure water $(18.2 \mathrm{M} \Omega \mathrm{cm}$, NANOpure Diamond, ThermoFisher, USA) was used throughout the test. All the reagents met the requirement of analytical grade.

The high-performance liquid chromatography (ICS3000, DIONEX, USA) system was combined directly to the inlet of the inductively coupled plasma mass spectrometer (ICP-MS) (X Series II type, Thermo Fisher, USA) for detection of arsenic speciation, including iAs ${ }^{\mathrm{III}}$, $\mathrm{iAs}^{\mathrm{V}}$, MMA and DMA. The mobile phase (20 mM ammonium carbonate) flowed through the anion exchange column at a rate of $1 \mathrm{~mL} / \mathrm{min}$ for no less than $10 \mathrm{~min}$ to obtain $\mathrm{pH}$ equilibration (2.0-3.5). The injection volume was $25 \mu \mathrm{L}$. With the time of sample injection, DMA, iAs ${ }^{\mathrm{III}}$, MMA and $\mathrm{iAs}{ }^{\mathrm{V}}$ were separated accordingly. Standard compounds containing iAs ${ }^{\mathrm{III}}$, iAs ${ }^{\mathrm{V}}$, MMA and DMA as mentioned above were used for calibration. The test of arsenic speciation was conducted at the Analysis Center of Tsinghua University, Beijing, China.

\section{SNPs of AS3MT gene \\ DNA extraction}

Blood samples were taken from 70 to 112 patients with informed consent of genotyping study. The karyocytes were separated out with red blood cell lysis buffer. The QIAamp DNA mini kit (Qiagen, Chatworth, CA) was used for DNA extraction from the karyocytes.

\section{Genotyping}

A total of 18 SNPs (T3963C, A4602G, T4740C, A6144T, G7395A, A9749G, A10209G, C12390G, T12590C, T14215C, T14458C, T25986C, T26790C, A27215G, T35587C, A35991G, C37616A, T37950C) were detected with polymerase chain reaction (PCR) method [13] (see Table 1 for the primer sequences of each genotype). Amplification was performed in a $25 \mu \mathrm{L}$ reaction mixture containing $0.2 \mu \mathrm{L}$ GoTaq (Promega, Madison, WI, USA), $5 \mu \mathrm{L} 5 \times$ Colorless GoTaq Reaction Buffer, $2 \mu \mathrm{L}$ dNTPs $(2.5 \mathrm{mM}), 1 \mu \mathrm{L}$ primer $(10 \mu \mathrm{M})$ and $2 \mu \mathrm{L}$ DNA sample $(30-100 \mathrm{ng} / \mu \mathrm{L})$. The PCR protocol consisted of initial denaturation at $96^{\circ} \mathrm{C}$ for $2 \mathrm{~min}$, followed by 35 cycles of denaturation at $95^{\circ} \mathrm{C}$ for $30 \mathrm{~s}$, annealing at $60{ }^{\circ} \mathrm{C}$ for 30 $\mathrm{s}$, and extension at $72{ }^{\circ} \mathrm{C}$ for $30 \mathrm{~s}$, and a final extension at $72{ }^{\circ} \mathrm{C}$ for $5 \mathrm{~min}$. The PCR products were then sent to Shanghai SimpleGene Clinical Laboratory for Sanger sequencing.

\section{Statistical analysis}

The concentration of TAs and arsenic speciation were documented in the form of "mean \pm standard deviation (SD)", as well as the percentage of different species of arsenic, and compared between groups by Student's $t$ test. While PMI and SMI were shown as "median (minimum-maximum)" because of non-normal distribution of the data, and compared between groups by Kolmogorov-Smirnov test. $P<0.05$ was determined for statistically significant difference. All statistical analyses were performed using SPSS 20.0 statistical package.

\section{Results}

The 112 patients were divided into different groups according to their time intervals between the cessation of ATO and the arsenic test, namely off ATO 0-6 months (0-6 months, 8 cases), $7-2$ months (10 cases), $1-2$ years 
Table 1 SNPs and primer sequence for PCR-based genotyping

\begin{tabular}{|c|c|c|c|c|}
\hline No. & Gene & Primer & Sequence & PCR size (bp) \\
\hline \multirow[t]{2}{*}{1} & 3963 (T to C) & 3963-F & AATGAATAGAAATGATCGTTACAAG & 377 \\
\hline & & $3963-R$ & ATGGAACACTTCACGAATTTGTATG & \\
\hline \multirow[t]{2}{*}{2} & 4602 (A to G) & $4602-F$ & CGAAGAAACTTGTGGGCCAGA & 260 \\
\hline & & $4602-R$ & TCGCTCCACTGCGATTTTCAC & \\
\hline \multirow[t]{2}{*}{3} & 4740 (T to C) & $4740-\mathrm{F}$ & CGAAGAAACTTGTGGGCCAGA & 223 \\
\hline & & $4740-R$ & CTGATTTAAATGAACACTCACCT & \\
\hline \multirow[t]{2}{*}{4} & 6144 (A to T) & $6144-F$ & GGTCACTAGGGAATTAACCCG & 414 \\
\hline & & $6144-R$ & CCAAGGTTGATTAGTGGGTGC & \\
\hline \multirow[t]{2}{*}{5} & 7395 (G to A) & $7395-\mathrm{F}$ & CGCCTATGGGACAGAAACCTT & 154 \\
\hline & & $7395-R$ & CTAAGGGACAGAGTGAGACTC & \\
\hline \multirow[t]{2}{*}{6} & 9749 (A to G) & 9749-F & CCAGACCAGCTTGAACAACATGGC & 388 \\
\hline & & 9749-R & CCCCAAGATCAGAGACAGAGT & \\
\hline \multirow[t]{2}{*}{7} & 10209 (A to G) & 10209-F & AAAGAGAGGAGGGAGGCGGTA & 291 \\
\hline & & 10209-R & GAGCTCTTAGTTCAAGGGGGA & \\
\hline \multirow[t]{2}{*}{8} & 12390 (C to G) & 12390-F & TTTAGGAGACAGACACTCTTAGAATG & 321 \\
\hline & & $12390-\mathrm{R}$ & ACTTTGTGTGTCCTGATTTCTTCTG & \\
\hline \multirow[t]{2}{*}{9} & 12590 (T to C) & $12590-F$ & GTTTCAGCATGGTGGGGAGTT & 172 \\
\hline & & $12590-R$ & AGCCTCATCCTGGCTATTAGC & \\
\hline \multirow[t]{2}{*}{10} & 14215 (T to C) & $14215-F$ & CTGTACAATGGTAACCCCCCA & 101 \\
\hline & & $14215-R$ & GCATGTCATCAGTTATCTTTCT & \\
\hline \multirow[t]{2}{*}{11} & 14458 (T to C) & 14458-F & GTGCTGGAGATGAACCGTGAA & 232 \\
\hline & & $14458-R$ & GCAAGGGCAAGAGCAGAAAGA & \\
\hline \multirow[t]{2}{*}{12} & 25986 (T to C) & 25986-F & СTTCTGGGCATTTTTTTCTTCTA & 236 \\
\hline & & 25986-R & GTTCTCTCAGGTAGTGAAAGCC & \\
\hline \multirow[t]{2}{*}{13} & 26790 (T to C) & 26790-F & TGATCGCCTGACATTCCTGGT & 203 \\
\hline & & $26790-R$ & CCAAGTGTCTCCAATTGTCCTG & \\
\hline \multirow[t]{2}{*}{14} & 27215 (A to G) & 27215-F & GCCTATCTGGCCAAACTCTTG & 221 \\
\hline & & 27215-R & CATGTTACCCAGGCTGGTGTC & \\
\hline \multirow[t]{2}{*}{15} & 35587 (T to C) & 35587-F & CAGCAGTCTTGTCTTTTAAAT & 188 \\
\hline & & 35587-R & GCTAAAACGACTTCTCTTCCC & \\
\hline \multirow[t]{2}{*}{16} & 35991 (A to G) & 35991-F & CACGTGCAAATGACAACCCCA & 227 \\
\hline & & $35991-R$ & GTTTGATTTAGGTTGACTTACA & \\
\hline \multirow[t]{2}{*}{17} & $37616(\mathrm{C}$ to $\mathrm{A})$ & 37616-F & GCCAAATCATGTTTGGTAGGAGG & 370 \\
\hline & & $37616-R$ & GTGCAGTGGTGCAATCATAGC & \\
\hline \multirow[t]{2}{*}{18} & 37950 (T to C) & 37950-F & CATGGTGAGACCCCCATCTCT & 462 \\
\hline & & $37950-R$ & CCAGCTCCTGATGATAATGACC & \\
\hline
\end{tabular}

(17 cases), 2-3 years (12 cases), 3-4 years (6 cases), 4-5 years (6 cases), 5-6 years (13 cases), 6-7 years (10 cases), 7-8 years (14 cases), 8-9 years (13 cases) till 9-10 years (3 cases). Seven positive controls were tested at 4 time points: d0, d7, CR and Con. The trend of TAs accumulation and excretion in plasma, urine, hair and nails had been previously reported (black curves in Fig. 1) [5]. To be brief, TAs in plasma and urine were excreted quickly to normal levels within 0 to 6 months, no higher than that of the healthy controls, but in hair and nails the excretion was delayed till after 6 months off ATO. Therefore, when comparing the long-term TAs and arsenic speciation in hair and nails, patients who had finished the ATO treatment for less than 6 months were excluded from the calculation.

\section{Arsenic speciation}

The trend of average concentration of iAs ${ }^{\mathrm{III}}$, iAs ${ }^{\mathrm{V}}$, MMA and DMA in patients' plasma, urine, hair and nails is also shown in Fig. 1. When patients were undergoing ATO treatment, arsenic speciation in their plasma demonstrated that the main component of TAs 

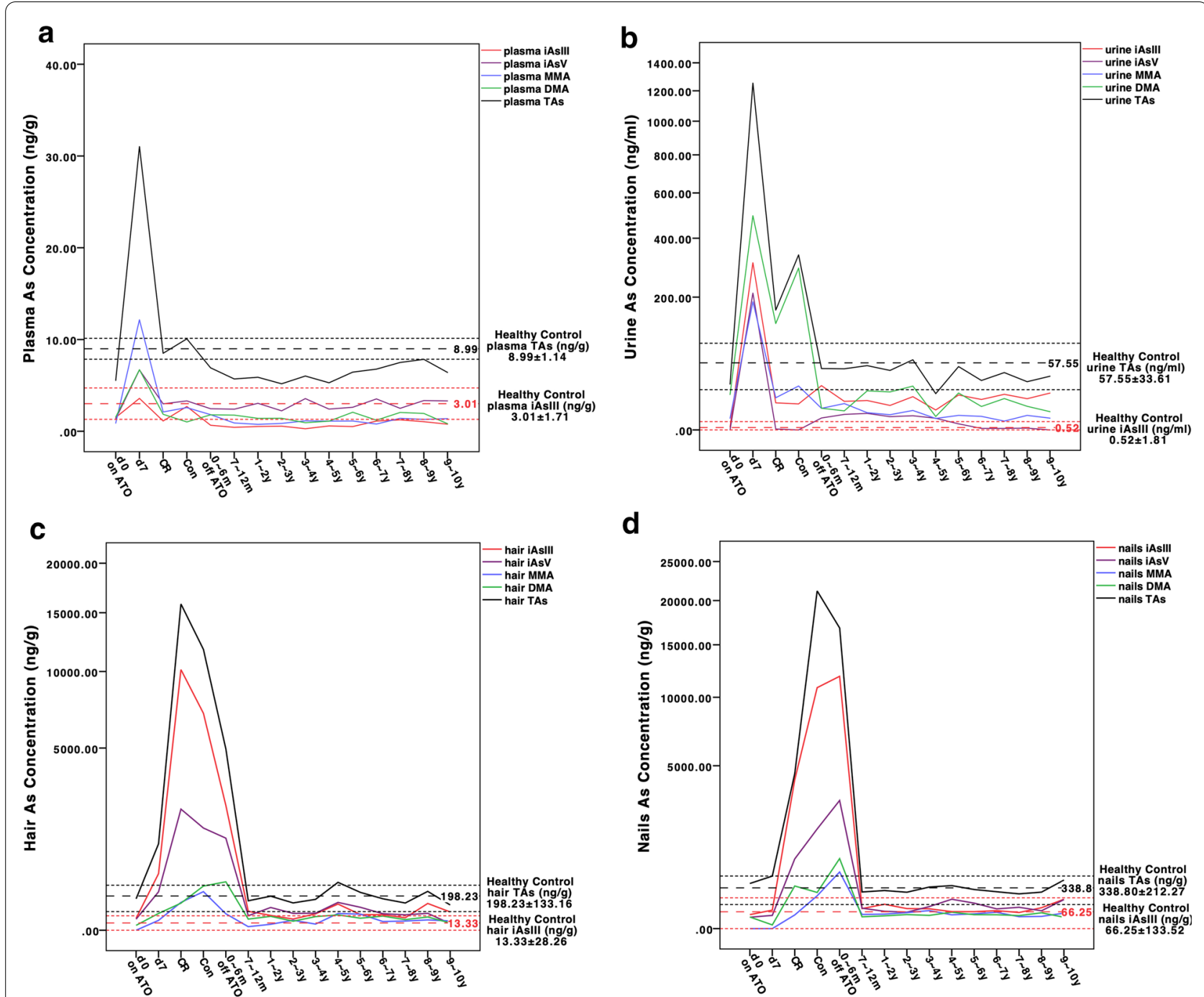

Fig. 1 TAs and arsenic speciation by time on and off ATO in patients' plasma (a), urine (b), hair (c) and nails (d). TAs and arsenic speciation were pointed at mean value for each group. The reference lines in black and red were mean \pm Standard Deviation (SD) for TAs and iAs ${ }^{\prime \prime \prime}$ of the healthy controls, respectively. TAs: total arsenic; ATO: arsenic trioxide; iAs'II: inorganic arsenite

was organic arsenic MMA and DMA, but not iAs ${ }^{\mathrm{III}}$ as in ATO (Fig. 1a), indicating that the methylation capacity of patients was sufficient to metabolize ATO during continuous infusion, and excessive iAs ${ }^{\mathrm{III}}$ was quickly excreted through the urine since iAs ${ }^{\mathrm{III}}$ was the second highest component of TAs in the urine during ATO treatment, second only to DMA (Fig. 1b). Therefore, the concentration of iAs ${ }^{\mathrm{III}}$ was relatively low in patients' blood, whereas higher in their urine. Meanwhile, due to high affinity of $-\mathrm{SH}$ in hair and nails to iAs ${ }^{\mathrm{III}}$, it was the main component of TAs in patients' hair and nails (Fig. 1c, d) during the treatment, and decreased till 6 months off ATO, which was consistent with the trend of TAs in hair and nails.
However, long-term follow-up showed that the percentage of iAs ${ }^{\mathrm{III}}$ in patients' urine, hair and nails were still significantly higher than the healthy controls, with an average of $40.08 \pm 28.90 \%$ vs. $1.94 \pm 7.19 \%(P<0.001)$ in urine, $29.25 \pm 16.44 \%$ vs. $13.29 \pm 26.54 \%(P=0.002)$ in hair, and $30.21 \pm 16.70 \%$ vs. $13.64 \pm 30.55 \%$ in nails $(P=0.003)$ (Table 2). The patterns of arsenic composition in patients and healthy controls were illustrated more specifically in Fig. 2. Obviously in urine samples (Fig. 2b) it was the most significantly different, with organic arsenic reaching over $80 \%$ (78.92\% of DMA and $2.49 \%$ of MMA) in the healthy controls, while iAs counting nearly a half $\left(40.08 \%\right.$ of $\mathrm{iAs}^{\mathrm{III}}$ and $8.72 \%$ of $\left.^{\mathrm{iAs}} \mathrm{V}^{\mathrm{V}}\right)$ in the patients off ATO (Table 2). Since the recent intake of iAs, which 
Table 2 TAs and arsenic speciation of patients and healthy control

\begin{tabular}{|c|c|c|c|c|c|c|c|}
\hline & TAs (ng/g) & $\mathrm{iAs}^{\mathrm{III}} \%$ & $\mathrm{iAs}^{\mathrm{v}_{\%}}$ & MMA\% & DMA\% & PMI & SMI \\
\hline \multicolumn{8}{|l|}{ Plasma } \\
\hline Patients & $6.43 \pm 1.56$ & $11.40 \pm 9.98$ & $44.47 \pm 19.81$ & $17.19 \pm 13.39$ & $24.50 \pm 15.47$ & $0.25(0-5.04)$ & $1.75(0.06-37.14)$ \\
\hline Control & $8.99 \pm 1.14$ & $33.87 \pm 18.92$ & $25.26 \pm 16.56$ & $19.29 \pm 14.53$ & $16.13 \pm 13.03$ & $0.28(0-8.74)$ & $0.82(0-19.2)$ \\
\hline$P$ value & $<0.001$ & $<0.001$ & $<0.001$ & 0.28 & $<0.001$ & 0.742 & 0.002 \\
\hline \multicolumn{8}{|l|}{ Urine } \\
\hline Patients & $45.28 \pm 33.28$ & $40.08 \pm 28.90$ & $8.72 \pm 11.42$ & $15.64 \pm 14.05$ & $26.36 \pm 26.64$ & $0.27(0-5.52)$ & $1.21(0-143.01)$ \\
\hline Control & $57.55 \pm 33.61$ & $1.94 \pm 7.19$ & $1.37 \pm 6.31$ & $2.49 \pm 3.52$ & $78.92 \pm 18.79$ & $0.27(0-1.24)$ & $16.36(3.32-120.34)$ \\
\hline$P$ value & 0.009 & $<0.001$ & $<0.001$ & $<0.001$ & $<0.001$ & 0.001 & $<0.001$ \\
\hline \multicolumn{8}{|l|}{ Hair } \\
\hline Patients & $195.43 \pm 202.25$ & $29.25 \pm 16.44$ & $34.26 \pm 20.82$ & $12.05 \pm 12.95$ & $18.87 \pm 11.95$ & $0.13(0-2.16)$ & $1.35(0-91.46)$ \\
\hline Control & $198.23 \pm 133.16$ & $13.29 \pm 26.54$ & $53.00 \pm 48.00$ & $14.93 \pm 35.01$ & $12.57 \pm 26.58$ & $0(0-0.21)$ & $0(0-0.01)$ \\
\hline$P$ value & 0.96 & 0.002 & 0.012 & 0.558 & 0.132 & 0.001 & 0.007 \\
\hline \multicolumn{8}{|l|}{ Nails } \\
\hline Patients & $294.65 \pm 158.94$ & $30.21 \pm 16.70$ & $32.85 \pm 18.08$ & $17.70 \pm 12.08$ & $15.72 \pm 10.14$ & $0.24(0-2.64)$ & $0.84(0-9)$ \\
\hline Control & $338.80 \pm 212.27$ & $13.64 \pm 30.55$ & $20.07 \pm 35.56$ & $8.71 \pm 26.27$ & $52.57 \pm 44.26$ & $0(0-0.45)$ & $0.35(0-0.69)$ \\
\hline$P$ value & 0.353 & 0.003 & 0.034 & 0.032 & $<0.001$ & 0.002 & 0.514 \\
\hline
\end{tabular}

TAs: total arsenic; iAs ${ }^{111} \%$ : inorganic arsenite/TAs $\times 100 \%$; iAs $\%$ : inorganic arsenate/TAs $\times 100 \%$; MMA\%: monomethylarsonic acid/TAs $\times 100 \%$; DMA\%: dimethylarsinic $\mathrm{acid} / \mathrm{TAs} \times 100 \%$; PMI: primary methylation index; SMI: secondary methylation index

was reflected by the sum of $\mathrm{iAs}^{\mathrm{III}}$ and $\mathrm{iAs}^{\mathrm{V}}$ in plasma (Fig. 2a), was no higher in patients, lower percentage of organic arsenic in the urine indicated insufficient arsenic methylation metabolism among patients.

Since liver dysfunction and hepatic steatosis were the main chronic AEs in the follow-up, we further evaluate the relationship between liver disorders and urine arsenic metabolism. Although the percentage of different species of arsenic was of no significant difference between patients with and without liver disorders, the urine PMI was lower in patients with liver dysfunction ( 0.37 vs. 0.59 , $P=0.047)$ and hepatic steatosis $(0.40$ vs. $0.68, P=0.027)$ compared to patients with normal liver examination (Fig. 3). It suggested that lower capacity of transforming iAs into MMA in patients might associate with more iAs accumulation in the long run and the consequently chronic liver toxicity.

\section{SNPs of $A S 3 M T$ gene}

The 18 SNPs of $A S 3 M T$ gene were genotyped among 70 patients. The concentration of urine TAs, the percentage of arsenic speciation, PMI and SMI were compared between the wild type and the mutation groups in each SNP. The data showed that two SNPs were associated with elevated urine SMI among patients (Table 3) when both mutated from allele A to G, with SMI 4.2 vs. $12.0(P=0.011)$ for A9749G, and 5.9 vs. 12.6 for A27215G $(P=0.017)$, indicating more sufficient methylation metabolism of arsenic. However, none of the 18 SNPs affected the urine PMI or chronic liver disorders according to the multivariant linear regression or logistic regression.

\section{Discussion}

The ATRA and ATO combination therapy for APL has brought significant advantages in both laboratory studies and clinical settings [1], especially when compared to the ATRA and anthracycline-based chemotherapy [3, $7,8]$, suggesting that the addition of arsenic in the frontline therapy would guarantee a longer remission duration and survival. However, ATO was still considered as a potential toxicant as well as a carcinogen, which was commonly observed after long-time exposure to environmental contamination $[18,19]$. In our previous study, the incidence of mild liver dysfunction (15.2\%) and hepatic steatosis $(42.9 \%)$ in patients was significantly higher compared to the healthy controls [5].

Toxicological studies have proved that the plasma and urine arsenic is related to the short-term arsenic exposure, while arsenic in hair and nails represents the long-term retention of iAs [10]. Urine arsenic speciation reveals the methylation capacity, with the least toxic DMA accounting for $60-80 \%$ of urine TAs. In our 5-year follow-up, Hu et al. [1] reported no significant TAs retention in patients' plasma, urine, hair and nails. While in this study the concentration of TAs in patients' plasma and urine was even lower than healthy controls (Table 2), indicating less arsenic exposure or intake from patients' recent environment. When arsenic speciation was taken into consideration, the metabolism, excretion and 


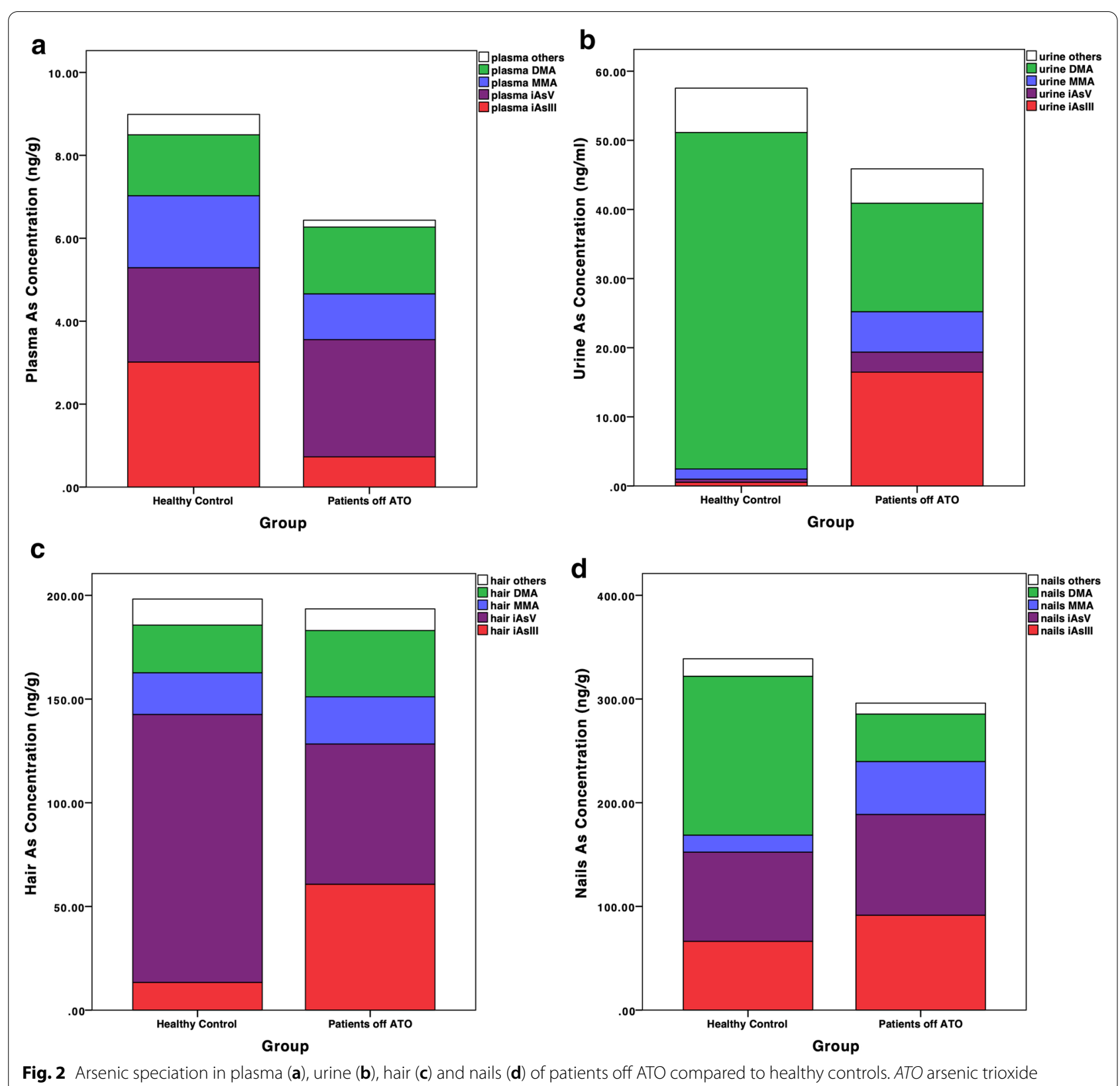

accumulation of ATO in patients' plasma, urine, hair and nails was profiled with more details. We reported a quick metabolism and excretion of iAs ${ }^{\mathrm{III}}$ in patients' plasma and urine right after the administration of ATO, while the accumulation of $\mathrm{iAs}{ }^{\mathrm{III}}$ in their hair and nails continued until 6 months after the cessation of ATO.

More importantly, the urine arsenic speciation revealed significantly different capacity of arsenic methylation between patients and healthy controls. The urine arsenic speciation in healthy controls was basically in line with the normal pattern of arsenic metabolism and distribution, with DMA reaching $78.92 \%$ of the urine TAs. On the contrary, the iAs ${ }^{\mathrm{III}} \%$ in patients' urine was significantly higher. The high level of unmethylated iAs ${ }^{\mathrm{III}}$ might accumulate in $-\mathrm{SH}$ enriched tissues and organs, which may be the cause of higher percentage of iAs ${ }^{\mathrm{III}}$ in patients' hair and nails even after 6 months off ATO, as well as ATO-associated chronic AEs. The impact of arsenic exposure on the methylation capacity was still not clear, but recent studies suggested that change of methylation capacity is dependent on the exposure time, concentration 

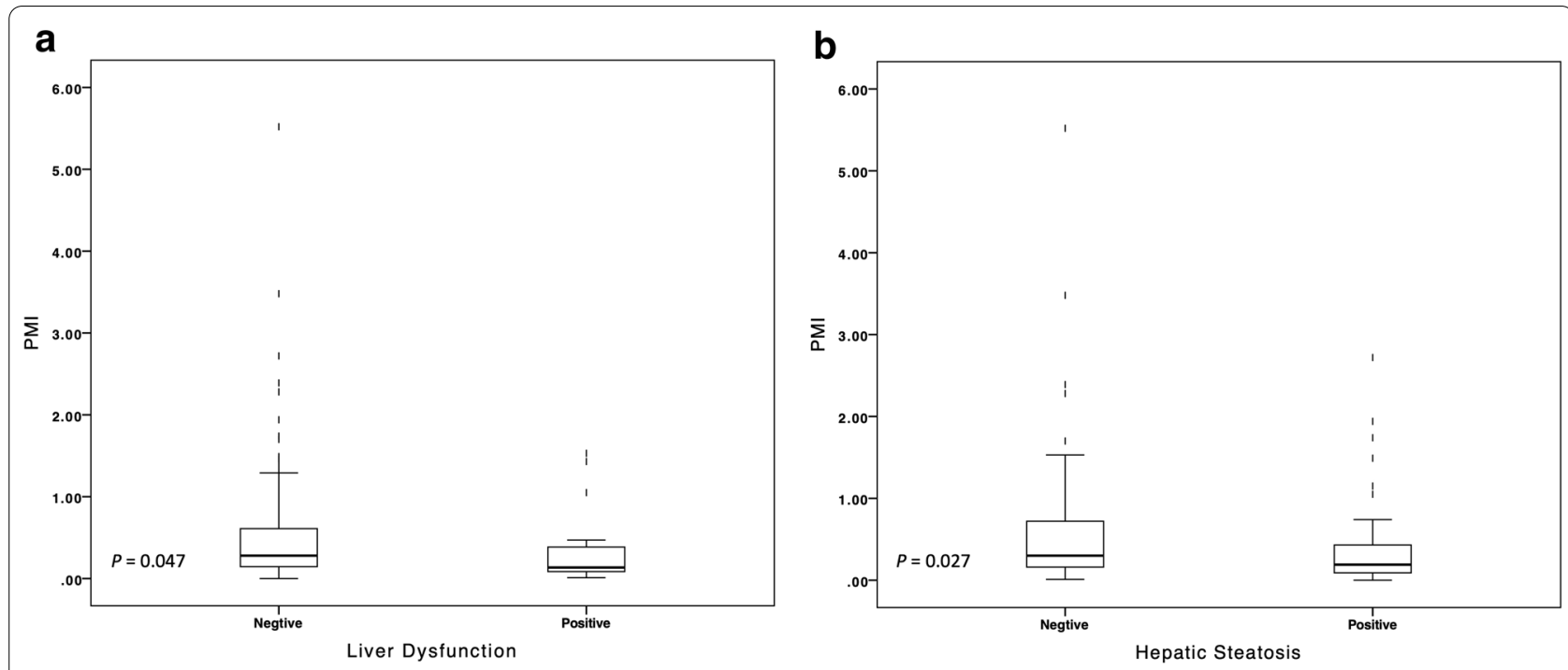

Fig. 3 The relationship of urine PMI with chronic liver dysfunction (a) and hepatic steatosis (b). PMI primary methylation index

Table 3 SNPs of AS3MT and the urine arsenic methylation metabolism

\begin{tabular}{|c|c|c|c|c|c|c|c|}
\hline $\mathrm{SNPs}^{\mathrm{a}}$ & TAs (ng/mL) & $i A s^{\prime \prime \prime} \%$ & $\mathrm{i} A s^{\mathbf{v}_{\%}} \%$ & MMA\% & DMA\% & PMI & SMI \\
\hline \multicolumn{8}{|l|}{9749} \\
\hline AA & $43.66 \pm 35.79$ & $41.16 \pm 31.22$ & $10.55 \pm 13.83$ & $16.54 \pm 13.44$ & $23.39 \pm 24.27$ & $0.21(0.02-2.39)$ & $1.05(0-41.17)$ \\
\hline$A G \& G G$ & $41.61 \pm 30.20$ & $29.04 \pm 30.13$ & $5.54 \pm 6.39$ & $5.96 \pm 3.64$ & $46.45 \pm 33.32$ & $0.21(0.02-1.33)$ & $7.5(0-29.06)$ \\
\hline$P$ value & 0.874 & 0.294 & 0.296 & 0.024 & 0.02 & 0.989 & 0.011 \\
\hline \multicolumn{8}{|l|}{27215} \\
\hline AA & $41.66 \pm 30.47$ & $43.23 \pm 30.00$ & $9.09 \pm 12.62$ & $15.69 \pm 13.04$ & $23.11 \pm 23.08$ & $0.20(0.02-2.39)$ & $0.96(0-143.01)$ \\
\hline$A G \& G G$ & $54.73 \pm 39.34$ & $23.73 \pm 27.78$ & $6.29 \pm 6.38$ & $9.42 \pm 14.73$ & $41.65 \pm 34.93$ & $0.26(0.02-1.53)$ & $6.41(0-41.17)$ \\
\hline$P$ value & 0.209 & 0.044 & 0.458 & 0.147 & 0.027 & 0.71 & 0.017 \\
\hline
\end{tabular}

SNP: single nucleotide polymorphism; AS3MT: (+ 3 oxidative state) methylation transferase; TAs: total arsenic; iAs ${ }^{\prime \prime \prime} \%$ : inorganic arsenite/TAs $\times 100 \%$; iAs $\%$ : inorganic arsenate/TAs $\times 100 \%$; MMA\%: monomethylarsonic acid/TAs $\times 100 \%$; DMA\%: dimethylarsinic acid/TAs $\times 100 \%$. PMI: primary methylation index; SMI: secondary methylation index

a All the other SNPs of AS3MT showed no significant influence on arsenic metabolism, therefore only A9749G and A27215G are presented

and the duration after arsenic exposure. Xu et al. [20] analyzed urine arsenic of patients with sub-acute arseniasis, chronic high-dose exposure and the controls, and reported that the sub-acute arseniasis group had significantly impaired methylation metabolism than the chronic high-dose group. Wei et al. [21] concluded that the arsenic methylation efficiency was significantly lower due to chronic exposure to high levels of arsenic in the environment. But Huang et al. [22] performed arsenic speciation for non-tumor residents in southern Taiwan with a 15-year interval after cessation of arsenic ingestion, and demonstrated an increase of methylation. In our study, the decreased capacity of methylation metabolism in patients seemed to continue even after 10 years off ATO.
The urine primary and secondary methylation indexes (PMI and SMI) simply reflect the methylation level of arsenic in human body. Studies have shown that lower urinary arsenic PMI or SMI might increase the risk of skin lesions, hypertension [18] and bladder cancer [23]. In our study, we showed that urine PMI was significantly lower in patients with liver dysfunction and hepatic steatosis, indicating that lower methylation capacity was associated with the incidence of chronic liver disorders. As we know, ATO-related acute liver dysfunction during the treatment of APL is common, with the incidence between 33 and 75\% [1, 24]. Lo-Coco et al. [3] reported that the incidence of grade 3-4 liver dysfunction in the ATRA + ATO group was even up to $57 \%$. When ATO is binding to the $-\mathrm{SH}$, it will produce more intracellular 
reactive oxygen species and lead to cytochrome $\mathrm{C}$ leakage, DNA damage and apoptosis [25]. More importantly, acute liver dysfunction might lead to a decrease in the capacity of arsenic methylation [26], thus relatively higher $\mathrm{iAs}{ }^{\mathrm{III}} \%$ in the urine, hair and nails would last for a long time as shown in our study. Incomplete methylation of iAs ${ }^{\text {III }}$ would cause tissue and organ damage under oxidative stress, including chronic liver dysfunction and hepatic steatosis as presented in our previous study. Santra et al. [27] reported that the fatty infiltration of liver in mice feeding with iAs was not observed until the 12th month, and liver fibrosis till the 15th month. Mazumder et al. [28] underwent liver biopsy in 69 patients in India diagnosed as arseniasis due to polluted drinking-water, and 63 of them presented non-cirrhosis liver fibrosis, indicating that the acute apoptosis of hepatocytes was likely to develop into chronic liver dysfunction manifested as fatty infiltration and fibrosis. Though intravenously administrated ATO might also cause chronic liver disorders in a similar way, there was no liver fibrosis documented yet in our follow-up study. Of note, almost a half of our patients enrolled in the follow-up study had hepatic steatosis, which might affect the capacity of arsenic methylation. So continuous monitoring of urine PMI during and after the treatment of ATO may predict the risk of chronic AEs caused by ATO. Besides, we should be cautious that these patients with hepatic steatosis might develop into non-cirrhosis liver fibrosis in case of further arsenic exposure, thus long-term clinical observation is warranted.

In 2001 Lin et al. [12] discovered an $S$-adenosyl methionine (SAM) dependent arsenic ( +3 oxidative state) methylation transferase (AS3MT) in the hepatocyte. Since then, a series studies have proved the importance of AS3MT in the arsenic methylation metabolism. The transformation of $\mathrm{iAs}^{\mathrm{III}}$ into MMA and DMA is mainly catalyzed by AS3MT. AS3MT polymorphism has been reported in several studies. Different genotypes of AS3MT gene produced different phenotypes of AS3MT enzymes, leading to variation in the methylation capacity and the metabolites of urine arsenic. The research into the genotyping for the original inhabitants with or without arsenic contamination showed that inhabitants living in the highly contaminated areas for thousand years tended to express more powerful genotypes of $A S 3 M T$ with stronger methylation capacity [29]. Some SNPs on the exons or introns of $A S 3 M T$ gene were related to individual's differences in the ability of arsenic metabolism. The best clarified was that the M287T heterozygote on the exons, which was associated with increased activity of AS3MT enzymes, more organic arsenic in the urine and higher methylation capacity [13]. However in Asian population, the M287T mutation was fairly rare [30]. In our study, the M287T heterozygote (T14458C) were detected only in 3 out of 70 patients, with no significant influence on the arsenic metabolism as measured by arsenic speciation in patients' urine. Other SNPs including G7395A, G12390C, G12390T, T35587C, T14215C, G35991A, which were considered to have more sufficient arsenic methylation in the urine [31,32], were also not noticed in this study. However, both A9749G and A27215G showed significant influence on the urine SMI, indicating a more sufficient arsenic methylation metabolism. It may help explain the variant incidence of acute AEs when patients are treated with ATO, which was also reported in Lu et al's study [15] that patients with both 35991 (rs10748835) AA and 35587 (rs11191453) TC + CC genotypes had the highest DMA\% and SMI, but the lowest $\mathrm{iAs} \%$, serum alanine aminotransferase and aspartate aminotransferase level, indicating that additive effects exist on arsenic metabolism and liver function. Nevertheless, none of the 18 SNPs was related to decreased PMI and chronic liver disorders in our study. Other SNPs or genes should be explored to better understand the changes in arsenic methylation capacity and the pathological process of chronic AEs among APL patients.

\section{Conclusions}

In summary, ATO was generally safe with no obvious TAs retention for patients with APL, but the long-term follow-up of arsenic speciation revealed an impaired methylation capacity with higher iAs\% in their urine, hair and nails after the cessation of ATO treatment, which might be associatedwith the occurrence of chronic liver disorders, as indicated by lower urine PMI. Two SNPs (A9749G and A27215G) of the AS3MT gene might be responsible for a more sufficient arsenic methylation metabolism with higher urine SMI. Urine PMI could be a monitoring index for chronic AEs of ATO, and the SNPs of AS3MT gene should be considered when determining the treatment dosage of ATO. The mechanisms for impaired capacity of arsenic methylation and chronic liver disorders are yet to be discovered.

\section{Abbreviations \\ ATO: Arsenic trioxide; APL: Acute promyelocytic leukemia; ATRA: All-trans retinoic acid; CR: Complete remission; OS: Overall survival; TAs: Total arsenic; iAs: Inorganic arsenics; MMA: Monomethylarsonic acid; DMA: Dimethylarsinic acid; AS3MT: Arsenic (+3 oxidative state) methylationtransferase; SNPs: Single nucleotide polymorphisms; AEs: Adverse effects; PCR: Polymerase chain reac- tion; PMI: Primary methylation index; SMI: Secondary methylation index.}

\section{Acknowledgements}

The authors are grateful to Zhi Xing and Xiu Huang (Analysis Center of Tsinghua University) for the technical assistance of arsenic tests.

\section{Authors' contributions}

Contribution: $\mathrm{H}-\mathrm{MZ}$ and J-ML designed the research and served as the principle investigators; $\mathrm{H}-\mathrm{MZ}, \mathrm{X}-\mathrm{YL}$ and JH designed the protocol for arsenic tests; 
YZ, Y-FM, H-JZ, LC, L-NW and Y-XZ carried out the arsenic tests; YZ, Y-FM, H-JZ and $\mathrm{H}-\mathrm{MZ}$ collected and interpreted the data; $\mathrm{H}-\mathrm{MZ}, \mathrm{X}-\mathrm{YL}$ and J-ML carried out the statistical analysis; $Y Z, Y-F M, H-J Z$ and $\mathrm{H}-\mathrm{MZ}$ wrote the manuscript; and all the authors contributed to the final draft. All authors read and approved the final manuscript.

\section{Funding}

This work was supported by grants from the National Natural Science Foundation of China (81870110 of H.-M. Z.; 81770144 of J.-M.L.; 81800141 of L.C.), and the Pilot Project of Chinese and Western Medicine-Clinical Collaboration for Major Difficult Diseases (J.-M.L.).

\section{Availability of data and materials}

The datasets used and analyzed during the current study are available from the corresponding author on reasonable request.

\section{Ethics approval and consent to participate}

The study was proved by the Ethics Committee of Ruijin Hospital in Shanghai, China (Approval Number 2012-48 and 2013-22)

\section{Consent for publication}

Not applicable.

\section{Competing interests}

The authors declare that they have no competing interests.

Received: 30 December 2020 Accepted: 27 January 2021 Published online: 06 February 2021

\section{References}

1. Hu J, Liu YF, Wu CF, Xu F, Shen ZX, Zhu YM, et al. Long-term efficacy and safety of all-trans retinoic acid/arsenic trioxide-based therapy in newly diagnosed acute promyelocytic leukemia. Proc Natl Acad Sci USA. 2009;106:3342-7.

2. Ravandi F, Estey E, Jones D, Faderl S, O'Brien S, Fiorentino J, et al. Effective treatment of acute promyelocytic leukemia with all-trans-retinoic acid, arsenic trioxide, and gemtuzumab ozogamicin. J Clin Oncol. 2009;27:504-10.

3. Lo-Coco F, Avvisati G, Vignetti M, Thiede C, Orlando SM, lacobelli S, et al. Retinoic acid and arsenic trioxide for acute promyelocytic leukemia. N Engl J Med. 2013;369:111-21

4. Iland HJ, Collins M, Bradstock K, Supple SG, Catalano A, Hertzberg M, et al. Use of arsenic trioxide in remission induction and consolidation therapy for acute promyelocytic leukaemia in the Australasian Leukaemia and Lymphoma Group (ALLG) APML4 study: a non-randomised phase 2 trial. Lancet Haematol. 2015;2:e357-66.

5. Zhu H, Hu J, Chen L, Zhou W, Li X, Wang L, et al. The 12-year follow-up of survival, chronic adverse effects, and retention of arsenic in patients with acute promyelocytic leukemia. Blood. 2016;128:1525-8.

6. Abaza Y, Kantarjian H, Garcia-Manero G, Estey E, Borthakur G, Jabbour E, et al. Long-term outcome of acute promyelocytic leukemia treated with all-transretinoic acid, arsenic trioxide, and gemtuzumab. Blood. 2017;129:1275-83.

7. Platzbecker U, Avvisati G, Cicconi L, Thiede C, Paoloni F, Vignetti M, et al. Improved outcomes with retinoic acid and arsenic trioxide compared with retinoic acid and chemotherapy in non-high-risk acute promyelocytic leukemia: final results of the randomized Italian-German APL0406 trial. J Clin Oncol. 2017:35:605-12

8. Russell N, Burnett A, Hills R, Betteridge S, Dennis M, Jovanovic J, et al. Attenuated arsenic trioxide plus ATRA therapy for newly diagnosed and relapsed APL: long-term follow-up of the AML17 trial. Blood. 2018;132:1452-4.

9. Gebel TW. Arsenic methylation is a process of detoxification through accelerated excretion. Int J Hyg Environ Health. 2002;205:505-8.

10. Watanabe T, Hirano S. Metabolism of arsenic and its toxicological relevance. Arch Toxicol. 2013:87:969-79.

11. Torres-Sanchez L, Lopez-Carrillo L, Rosado JL, Rodriguez VM, Vera-Aguilar $\mathrm{E}$, Kordas K, et al. Sex differences in the reduction of arsenic methylation capacity as a function of urinary total and inorganic arsenic in Mexican children. Environ Res. 2016:151:38-43.

12. Lin S, Shi Q, Nix FB, Styblo M, Beck MA, Herbin-Davis KM, et al. A novel S-adenosyl-L-methionine:arsenic(III) methyltransferase from rat liver cytosol. J Biol Chem. 2002;277:10795-1803.
13. Fujihara J, Soejima M, Yasuda T, Koda Y, Agusa T, Kunito T, et al. Global analysis of genetic variation in human arsenic ( +3 oxidation state) methyltransferase (AS3MT). Toxicol Appl Pharmacol. 2010;243:292-9.

14. Lu J, Hu S, Wang W, Li J, Dong Z, Zhou J, et al. AS3MT polymorphisms, arsenic metabolism, and the hematological and biochemical values in APL patients treated with arsenic trioxide. Toxicol Sci. 2018;166:219-27.

15. Lu J, Yu K, Fan S, Liu W, Dong Z, Li J, et al. Influence of AS3MT polymorphisms on arsenic metabolism and liver injury in APL patients treated with arsenic trioxide. Toxicol Appl Pharmacol. 2019:379:114687.

16. Liu WS, Wang XY, Lu J, Zhang YM, Ye XM, Li JM, et al. Polymorphisms in arsenic ( +3 oxidation state) methyltransferase (AS3MT) predict the occurrence of hyperleukocytosis and arsenic metabolism in APL patients treated with $\mathrm{As}_{2} \mathrm{O}_{3}$. Arch Toxicol. 2020;94:1203-13.

17. Shen ZX, Shi ZZ, Fang J, Gu BW, Li JM, Zhu YM, et al. All-trans retinoic acid $/ \mathrm{As}_{2} \mathrm{O}_{3}$ combination yields a high quality remission and survival in newly diagnosed acute promyelocytic leukemia. Proc Natl Acad Sci USA. 2004; 101:5328-35.

18. Wei B, Yu J, Wang J, Yang L, Li H, Kong $C$, et al. The relationships between arsenic methylation and both skin lesions and hypertension caused by chronic exposure to arsenic in drinking water. Environ Toxicol Pharmacol. 2017:53:89-94.

19. Huang HW, Lee $\mathrm{CH}$, Yu HS. Arsenic-induced carcinogenesis and immune dysregulation. Int J Environ Res Public Health. 2019;16(15):2746.

20. Xu Y, Wang Y, Zheng Q, Li B, Li X, Jin Y, et al. Clinical manifestations and arsenic methylation after a rare subacute arsenic poisoning accident. Toxicol Sci. 2008;103:278-84.

21. Wei B, Yu J, Kong C, Li H, Yang L, Guo Z, et al. An investigation of the health effects caused by exposure to arsenic from drinking water and coal combustion: arsenic exposure and metabolism. Environ Sci Pollut Res Int. 2017;24:25947-54.

22. Huang YK, Huang YL, Hsueh YM, Wang JT, Yang MH, Chen CJ. Changes in urinary arsenic methylation profiles in a 15-year interval after cessation of arsenic ingestion in southwest Taiwan. Environ Health Perspect. 2009;117:1860-6.

23. Chen YC, Su HJ, Guo YL, Hsueh YM, Smith TJ, Ryan LM, et al. Arsenic methylation and bladder cancer risk in Taiwan. Cancer Causes Control. 2003; $14: 303-10$

24. Lengfelder $\mathrm{E}$, Hofmann WK, Nowak D. Impact of arsenic trioxide in the treatment of acute promyelocytic leukemia. Leukemia. 2012;26:433-42.

25. Dai J, Weinberg RS, Waxman S, Jing Y. Malignant cells can be sensitized to undergo growth inhibition and apoptosis by arsenic trioxide through modulation of the glutathione redox system. Blood. 1999:93:268-77.

26. Wang H, Xi S, Liu Z, Yang Y, Zheng Q, Wang F, et al. Arsenic methylation metabolism and liver injury of acute promyelocytic leukemia patients undergoing arsenic trioxide treatment. Environ Toxicol. 2013;28:267-75.

27. Santra A, Maiti A, Das S, Lahiri S, Charkaborty SK, Mazumder DN. Hepatic damage caused by chronic arsenic toxicity in experimental animals. J Toxicol Clin Toxicol. 2000;38:395-405

28. Mazumder DN. Effect of chronic intake of arsenic-contaminated water on liver. Toxicol Appl Pharmacol. 2005;206:169-75.

29. Schlebusch CM, Lewis CM Jr, Vahter M, Engstrom K, Tito RY, Obregon-Tito AJ, et al. Possible positive selection for an arsenic-protective haplotype in humans. Environ Health Perspect. 2013:121:53-8.

30. Fujihara J, Soejima M, Koda Y, Kunito T, Takeshita H. Asian specific low mutation frequencies of the M287T polymorphism in the human arsenic ( +3 oxidation state) methyltransferase (AS3MT) gene. Mutat Res. 2008;654:158-61.

31. Schlawicke Engstrom K, Broberg K, Concha G, Nermell B, Warholm M, Vahter M. Genetic polymorphisms influencing arsenic metabolism: evidence from Argentina. Environ Health Perspect. 2007;115:599-605.

32. Meza MM, Yu L, Rodriguez YY, Guild M, Thompson D, Gandolfi AJ, et al. Developmentally restricted genetic determinants of human arsenic metabolism: association between urinary methylated arsenic and CYT19 polymorphisms in children. Environ Health Perspect. 2005:113:775-81.

\section{Publisher's note}

Springer Nature remains neutral with regard to jurisdictional claims in published maps and institutional affiliations. 